\title{
Application of Object Petri Net in the Modeling and Evaluation of Information Superiority
}

\author{
LU Cong $^{1, a}$, LING Yunxiang ${ }^{2,1, b}$, ZHANG Yaohong ${ }^{1}$ \\ ${ }^{1}$ Science and Technology on Information Systems Engineering Laboratory, National University of \\ Defense Technology, Changsha 410073, P.R. China \\ ${ }^{2}$ Department of Management Science and Engineering, Officers College of Chinese Armed Police \\ Force, Chengdu 610213, P.R. China \\ alucong_Ic@126.com, byxling@tom.com
}

Keywords: Object Petri Net; information superiority; modeling and evaluation

\begin{abstract}
Information is becoming increasingly important in modern warfare. Information superiority is an advantage in achievement and application of information of two hostile sides. It becomes the key of winning a battle obtaining information superiority and dominance. This paper introduces the basic knowledge of the modeling method of object Petri net and analyses its superiority on system modeling. It analyses the forming process of information superiority in detail and then gives the evaluation indexes. In the end, object Petri net is applied to the modeling and evaluation of information superiority. It builds up an overall application framework and analyses the applicability of the object Petri net method.
\end{abstract}

\section{Introduction}

With the rapid development of computer science and information technology, the importance of information ability becomes increasingly apparent on obtaining network resources, business competition and so on, especially in the field of military and national defense. As an important strategic resource, information has become the dominant factor affecting military operations, and also the "carrier" to measure and improve the combat capability such as military command, combat operations and fire attack. The ability to obtain information superiority become a key factor in winning the war.

Although the information superiority gets attention around the world and also the information technology develops by leaps and bounds, it still stages in the fog for most countries on whether one can obtain information superiority, how to judge, and how to make use. On the one hand, information superiority is not yet a very mature concept and it has not formed a comprehensive information space either. On the other hand, although researches on information and information superiority have been carried out, it still lacks a rational method and model to measure the effectiveness, especially lack of analysis model based on data.

Object Petri net has strong applicability and obvious superiority in researching and studying complex system of hierarchy structure, and also it has the strong ability of mathematical analysis. With object Petri net modeling tools, people can build up executable models, which can simulate the complex structure and interactive relationship automatically or interactively, record and do mathematical calculation of related data like communication flow, time and efficiency. With these advantages, object Petri net provides an effective evaluation method.

Currently, about information superiority and abilities, most of the studies do their evaluation from the point of view of the three indicators including completeness, correctness and currency. The related theoretical research and demonstration have been already plentiful and thorough. This paper will do some analysis for these three indicators, describe the modeling and simulation method of object Petri net and then apply it to the assessment of information superiority. An evaluation method and a model framework will be built up based on the object Petri net. 


\section{Brief Introduction to Object Petri Net}

Petri net was put forward by a German doctor named Carl Adam Petri in 1962, which is a kind of network used to describe the time and conditional relationship ${ }^{[1-2]}$. It uses four kinds of elements for system modeling: place, transition, arc and token. Petri net has its unique advantage in logic verification for that Petri net makes a combination of data flow, control flow and state transition, so it can naturally describe the features like concurrency, synchronization, resource contention, etc. Petri net has a self-control mechanism and gathers canonical representation and execution in the same model. It describes the static function and structure of a system by using place, transition and arc connection and describes the dynamic behavior of system with ignition of switch and transition of token. Petri net model will do the state transition automatically as long as meet the given conditions or constraints.

Object-oriented $^{[3]}$ technology is a kind of important idea in computer programming. The so-called object is a kind of software construction integrated with state (property) and behavior (operation), which is used to describe a physical or conceptual entity in real world. The basic idea of object-oriented is to achieve the system analysis and designing through the use of concept and mechanism like object, class, inheritance, abstraction, encapsulation, and so on. As named, Object-oriented modeling technology is using the concept and idea of object-oriented to model and analyse for systems. By giving the object different attributes and operations, the object-oriented modeling method presents the activity features of objects and maps it to computer system.

Object Petri net integrates Petri net and object-oriented modeling, the basic idea is mapping the target system into cooperative objects and using Petri net to describe the behavior of objects and the communication relationships between objects ${ }^{[4,5]}$. It introduces the concept and mechanism of object into the traditional Petri net. As the basic module, object is also the basic unit for model reusing, and actually it is a subnet encapsulated. Objects achieve interaction through interfaces while their internal structures are described by extended Petri net. Object Petri net makes up for the shortage of traditional one in that the information carried by place, transition and token is not rich enough and it lacks semantic description. In addition, Object Petri net adds the factor of time, so it is more suitable to describe the combat process and analyse the information superiority in the context of modern information war.

\section{Evaluation Indexes of Information Superiority}

A lot of researches and studies about information superiority have been carried out both in China and abroad to understand, explore and research the concept, theory and application of information superiority from different points of views ${ }^{[6-8]}$. Among them, report named "Exploring Information Superiority" [9] made by RAND Corporation does a comprehensive and embedded analysis for information superiority. It puts forward the $C^{4}$ ISR Information Superiority Reference Model (Fig.1). This model fixes out the related definition, features and attributes of key concepts from three different domains (Physical domain, Information domain and Cognitive domain), and also builds up the connections between them. In this way, it measures the degree of realization of information superiority and the guiding effects for military exercise. 


\section{Cognitive domain}

Situational awareness, shared situational awareness, collaboration, and decisionmaking

\begin{tabular}{|c|c|}
\hline $\begin{array}{l}\text { Prior knowledge } \\
\text { Expectations, concerns } \\
\text { Information domain }\end{array}$ & $\begin{array}{l}\text { Structured information } \\
\text { (CROP) }\end{array}$ \\
\hline \multicolumn{2}{|c|}{$\begin{array}{l}\text { Data collection, fusion to produce the CROP, dissemination of the CROP, } \\
\text { and sensor tasking }\end{array}$} \\
\hline $\begin{array}{c}\text { Collected } \\
\text { data }\end{array}$ & $\begin{array}{l}\text { Sensor } \\
\text { tasking }\end{array}$ \\
\hline Ground truth: entities, systems & tions, plans and physical activities \\
\hline
\end{tabular}

Figure 1 The Information Superiority Reference Model

The Information Superiority Reference Model showed in Fig. 1 describes the information flow relations of general $\mathrm{C}^{4} \mathrm{ISR}$ system, including process of information acquisition, fusion and distribution. Know then, the working process of command and control in fact is the process of information transformation. And this transformation process is consistent with the formation of the information superiority value chain. As a result, information superiority finally reflects in the quality of situation awareness produced by $\mathrm{C}^{4} \mathrm{ISR}$ system. Therefore, we can do the quantitative description for information superiority with the quality of information. As a matter of fact, there are lots of indexes to describe the information quality, and the choice and definition may be very different according to the integration or segmentation of the concept. Among these indexes, this paper mainly takes three into account which are most important and most accepted by researches: completeness, correctness and currency. The comprehensive evaluation of these three indicators can not only reflect the obtaining condition of information superiority, but also embody the ability to deal with information under the informational environment.

According to the research report of RAND Corporation, the three indexes of information superiority can be described as in Table 1 :

Table 1 Measures of Information Quality

\begin{tabular}{cl}
\hline Measure & \multicolumn{1}{c}{ Description } \\
\hline Completeness & $\begin{array}{l}\text { The degree to which the information is free of gaps with respect to the } \\
\text { relevant ground truth } \\
\text { Correctness }\end{array}$ \\
The degree to which the information agrees with ground truth \\
Currency & The time required to produce a CROP \\
\hline
\end{tabular}

The description of the three indexes in the table is suited to $C^{4}$ ISR system. However, in actual application, the conception of index differs up to the specific operational environment and mode. Considering the universality of application, here we make the three indexes for general description:

(1) Completeness: in the specified range of time and task, the degree of how the amount of perceived information meets the practical expected one, i.e.: 


$$
\frac{\text { the amount of perceived or obtained target information in specified range of time and task }}{\text { the amount of expected information in specified range of time and task }}
$$

Inside, the amount of target information can be the individual number of targets, the number of species, the number of parameters and such other conventional battlefield facts, but can also be the related information produced from the network like data flow and those similar ones. Therefore, data like probability of blind detection, probability of transmission loss can also be used to present the completeness.

(2) Correctness: the degree of consistency of the perceived information and the practical information.

$$
V(t)=\frac{\text { the amount of perceived information that agree with the truth in specified range of time and task }}{\text { the amount of obtained information in specified range of time and task }}
$$

The measurement of correctness can not only be the comparison of the actual numerical value such as color, number and so on, but also can be the fitting degree of data, and in the latter condition, the description of correctness is relatively indirect.

(3) Currency: the time required to form situation awareness from the moment when the target actions begin.

Currency is a measurement of time, which involves many factors like communication delay, command and decision efficiency, computing performance, etc.

\section{The Modeling and Evaluation Method Based On Object Petri Net}

Formation of Information Superiority. What information superiority reflects is the acquisition and processing ability of information. The purpose of information superiority modeling and evaluation is to do modeling and simulation for the series process of information acquisition and processing with specific modeling tools and get the experimental results and data. Through the statistical analysis of recorded data, we can measure the corresponding information-processing method and the superiority ability of the system from the evaluation indexes.

In the information warfare environment, suppose considering the information superiority ability of Red team, it is substantially to establish the procedural model from the information source to formation of situation awareness of Red team. The conceptual framework shows as Fig.2.

As showed in Fig.2, setting the Blue side as the information source, the particular actions generate information and form an information space. This information space not only has public information like external environment, public parameters, but also includes hidden information, encrypted information, deceiving information and some other indirect information of Blue side. Therefore, the information superiority of Red side mainly embodies in that the red completes its information processing (including information obtaining, calculating, responding, etc.) from the information space as comprehensive and accurate as possible with the shortest time, which agrees with completeness, correctness and currency as described above. 


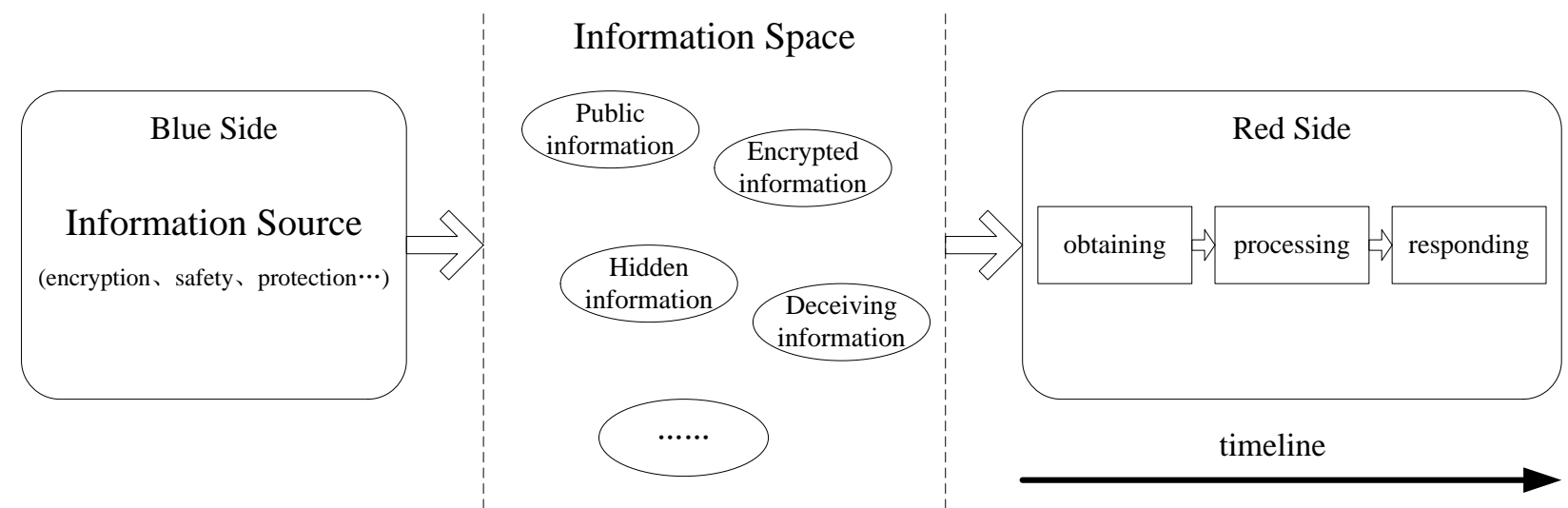

Figure 2 The Formation Framework of Information Superiority

The Modeling Train of Thought With Object Petri Net. As can be known from the formation of information superiority, the information process is not a single link or department. It exists in a large and complex system involving many operational nodes and related procedures. While modeling for such a system, object Petri net shows excellent applicability. The core of object Petri net is embedding a whole Petri net model as an independent object into the upper Petri network through interface.

The formation framework of information superiority showed in Figure 2 has in fact already formed the conceptual model of a confrontation. Corresponding to the data requirements of the evaluation indexes of information superiority, an overall framework of object Petri net application can be established as showed in Fig.3.

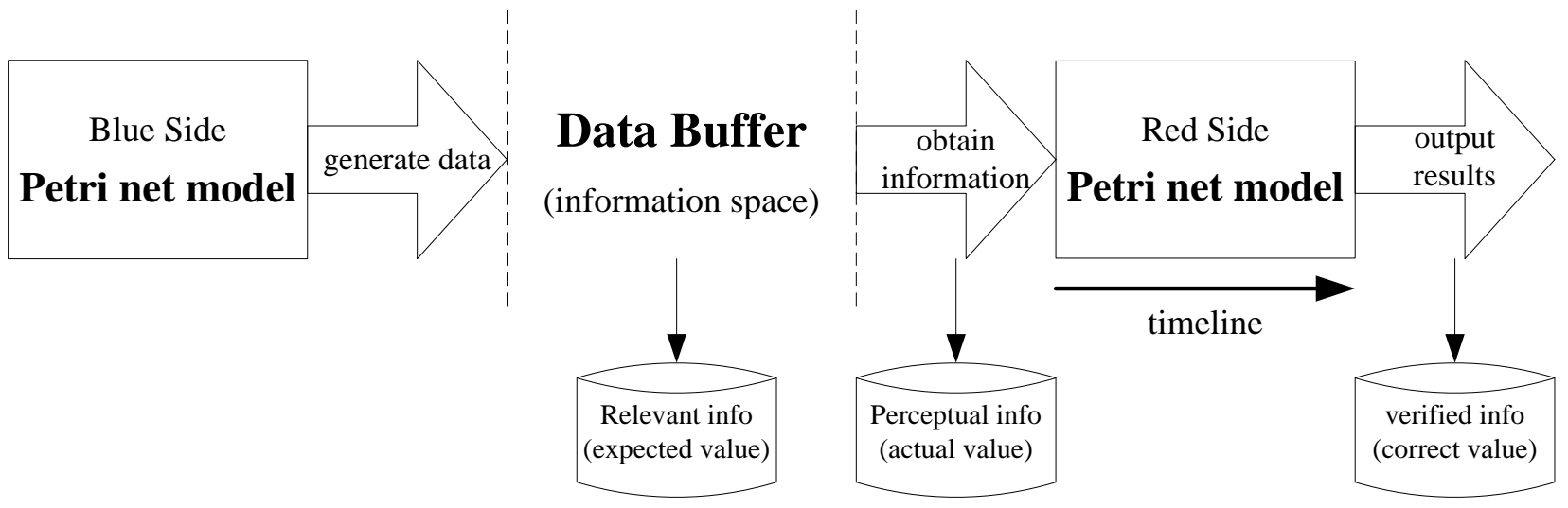

Figure 3 The Framework of Object Petri Net Modeling

An object Petri net model can be built up according to the framework of combat showed in Fig.3. During the modeling, three databases will be set up including database for total relevant information, database for perceptual information of one side, and database for those correct which has been verified. From these databases, data needed by the evaluation indexes of information superiority will be provided, with which the fitting function of time can be established. Then we get the condition of information superiority ability of the operation corresponding to the model. Specifically, it can be divided into three nested objects to create the object Petri net for either side:

Firstly, according to the understanding of the information processing, set every information-related task as a sub object to model the processes, refine the related task like information acquisition, data calculation and communication transmission, and then establish sub objects like information judge model, data fitting model, wire communication model, and others.

Afterwards, set each key node of combat as sub objects and use object Petri net to model operational process of each single department. During this modeling, the sub objects of information processing will be included and then the integrated process from accepting task to finishing it will be present. Such sub objects are those like model of radar, model of Command center, etc. 
Finally, set the whole operation process as the main object, use object Petri net to model the total operational activities and form the top-level model, during which the key node sub objects are applied.

When modeling with object Petri net, the analysis of the whole combat is executed from top to bottom to refine step by step, while the modeling procedure is bottom-up to create objects hierarchically. Among them, the top-level model (the main object) is mainly used to set the initialization of global properties, completing the definition of global properties and giving the initial value. The key node sub objects present the execution of the corresponding department or task especially the information link. The information processing sub objects specify the procedure of information acquisition, calculation and some other activities, and in terms of the evaluation of information superiority, this part is the absolute core of modeling, which mainly includes the writing and reading of the condition set and resource set of the global properties, the imitation of uncertainty, the output of simulation, and so on. Therefore, the modeling of information processing sub objects should restore the reality as comprehensive and accurate as possible.

Model Description Mechanism. The formation of information superiority involves many aspects such as relationship of command and cooperation, transformation of intelligence and information, control of time and opportunity, etc. Considering these actual situation, specific mechanism can be set up by object Petri net to handle with them.

(1) Mechanism of Data Transmission: Informatization is indeed data transferring in the network system, while in Petri net models, token has the convenience to simulate this. Token is capable of carrying many kind of information like data types, parameters, etc. Therefore, by setting the Token Property and depending on the transfer of Token between objects, the Petri net can reflect the obtaining and transmission of detected information, the uploading and downloading of orders or reports, and so on.

(2) Mechanism of Command and Control: Between combat departments, especially the superior-subordinate ones, there are complex command and control relationships. For instance, the execution unit will join the system task and take action only when receiving the orders of superiors. Such command and control can be solved by setting global variables or 0-1 identifications as its condition state, and the Petri network models judge these conditions in switches to realize the control function.

(3) Mechanism of Conflict Handling: Combat network is a task system with serial and parallel structures overlapped and interwoven. Meanwhile, the turnover of time information always causes conflict. Fortunately, there are always certain rules of treatment for different intelligence in combat. So in the object Petri net model, it can be done by setting priority for the switches to handle the conflict problems. Tasks and Objects with high priority will occupy the resource firstly to finish the activities like information communication.

(4) Mechanism of Breakdown and Delay Imitation: What concerns most in information war is confrontation activities like electromagnetic interference. So there are possibilities such as uncertainty, instrumental error, communication failure and so on. When modeling, it can be solved in some shift or switch of objects by using random number or calling for external function through components to imitate the error rate of detection, the loss rate of intelligence transmission and other similar probabilistic events. With local and global variables, it records the delay and efficiency of every single node and the whole process, and then data relate to information superiority will be obtained.

\section{Summary}

Information has become the dominant factor of the battlefield in information war and the importance of information superiority is increasingly apparent. Information superiority, with rich connotation, involving a broad range, is always throughout the large combat system. While, the modeling and simulation method of object Petri net can not only run within the top-down approach by stepwise refinement, but can also merge together one by one within the bottom-up approach. 
Therefore, object Petri net has great applicability for modeling and evaluation of information superiority.

This paper provides a detailed analysis for the concept and formation of information superiority and gives a general description of the evaluation indexes of information superiority. From the view of actual demand and the general process of information acquisition, processing and distribution, it puts forward the modeling and evaluation method for information superiority based on object Petri net, presents the research idea combined with object Petri net and information superiority and then verifies the support for the mathematical description of the evaluation indexes. In a certain sense, this may lay the foundation for the quantitative study of information superiority

\section{References}

[1] Carl Adam Petri. Communication with automata [D]. Darmstadt: Darmstadt University of Technology, 1962.

[2] YUAN Chongyi. The principle and application of Petri nets[M]. Beijing: Publishing House of Electronics Industry, 2005.

[3] WEN Dengmin. The theory and practice of object-oriented[M]. Chengdu: Southwest Jiaotong University Press,2005.

[4] Yue Xiaobo, Wang Lin, Tuo Qing. Research on Object-oriented Petri Net Modeling[J]. Computer Engineering, 2002,28(5):86.

[5] SHu Yuanzhong, Liu Yanpei, Peng Xiaohong, Chen ZHiyong. Survery on object-oriented Petri net modeling[J]. Computer Engineering and Design, 2010,31(15):3432.

[6] David S. Alberts, John J. Garstka, Frederick P. Stein. Network Centric Warefare: Developing and Leveraging Information Superiority(2nd Edition)[M]. CCRP, 1999.

[7] David S. Alberts, John J. Garstka, et al. Understanding Information Age Warefare[M]. Washington DC, CCRP Publication Series, 2001.

[8] Waltz E. Information Information Warfare: Principles and Operations. Boston London, USA. Artech House, 1998.

[9] Walter Perry, David Signori, John Boon. Exploring Information Superiority: A Methodology for Measuring the Quality of Information and Ins Impact on Shared Awareness[R]/RAND MR-1467, 2003.USA:Rand, 2003. 